\title{
ORDER IDEALS OF MINIMAL GENERATORS
}

\author{
E. GRAHAM EVANS AND PHILLIP GRIFFITH
}

\begin{abstract}
Let $R$ be a local noetherian domain with algebraically closed residue field and let $M$ be a finitely generated module of rank $r$ which is not free. Then there is some minimal generator $x$ of $M$ such that the ideal of images of $x$ under maps of $M$ to $R$ has height at most $r$.
\end{abstract}

Let $R$ be a local domain with maximal ideal $m$ and let $M$ be a finitely generated $R$-module. If $x$ is an element of $M$, then the order ideal of $x$, denoted $O_{M}(x)$, is defined to be the ideal of images of $x$ under homomorphisms of $M$ to $R$. In [4] Eisenbud and Evans showed that if $x$ is in $m M$, then the height of $O_{M}(x)$ is at most the rank of $M$. If $M$ is isomorphic to the free module $R^{n}$ and if $x$ in $M$ has components $x=\left\langle x_{1}, \ldots, x_{n}\right\rangle$, then $O_{M}(x)$ is the ideal generated by the $x_{i}$. Hence, in this case, the result follows from Krull's altitude theorem. The proof of this result given in [4] requires the assumption that $R$ possess a maximal Cohen-Macaulay module. Recently Bruns [2] has removed this assumption. The notion of order ideal provided a crucial step in our recent solution of the syzygy problem [5]. In that article we show that, if $R$ contains a field (in order that homomorphic images of $R$ have maximal Cohen-Macaulay modules), if $M$ is a $k$ th syzygy and if $x$ is a minimal generator of $M$ (that is, $x \in M-\mathfrak{m} M$ ), then the height of $O_{M}(x)$ is at least $k$. This provided the key step in showing that nonfree $k$ th syzygies have rank at least $k$. Our proof would have been more direct if we had shown that some minimal generator must have order ideal of height at most the rank. We note that this is the best one could expect, since modules of small rank can have minimal generators whose order ideals are proper but of large height. We give two methods of constructing such examples in $\S 2$. In $\S 1$, we show that, if the residue field is algebraically closed and if $M$ is not free, then some minimal generator has order ideal of height at most the rank of $M$. This allows a sharp dichotomy between nonfree modules of rank less than $k$ and $k$ th syzygies. For either the module has rank less than $k$ and hence some minimal generator has order ideal of height less than $k$, or $M$ is a $k$ th syzygy and the order ideal of every minimal generator has height at least $k$.

1. The main result. Let $(R, \mathfrak{m}, \mathfrak{f})$ be a local domain. Throughout this section we shall assume that $R$ is universally catenary and that $f$ is algebraically closed. The latter assumption is needed to insure that the maximal ideals of $R\left[X_{1}, \ldots, X_{t}\right]$ which contains the maximal ideal $m$ of $R$ have the form $\left(\mathfrak{m}, X_{1}-a_{1}, \ldots, X_{t}-a_{t}\right)$, where the $a_{i}$ are in $R$. We now state our main result.

Received by the editors April 30, 1981 and, in revised form, September 24, 1981.

1980 Mathematics Subject Classification. Primary 13C15; Secondary 14C99, 14F05. 
THEOREM. Let $(R, \mathfrak{m}, \mathfrak{f})$ be a universally catenary local domain with $\mathfrak{f}$ algebraically closed. Let $M$ be a finitely generated $R$-module such that the height of $O_{M}(x)$ exceeds the rank of $M$ for every minimal generator $x$ of $M$. Then $M$ is a free $R$-module.

The result follows immediately from the following lemma which is a more technical statement that describes precisely where the required minimal generators can be obtained. The notation $V(J)$ will denote the Zariski closed set of all prime ideals which contain $J$.

Lemma. Let $(R, \mathfrak{m}, \mathfrak{f})$ be a universally catenary local domain with $\mathfrak{f}$ algebraically closed. Let $M$ be a finitely generated nonfree $R$-module of rank $r$ with minimal generating set $e_{1}, \ldots, e_{t}$. Then there is a homogeneous ideal $J$ in $\mathfrak{f}\left[X_{1}, \ldots, X_{t}\right]$ of height at most $r$ such that to each maximal ideal $\left(X_{1}-a_{1}, \ldots, X_{t}-a_{t}\right)$ in $V(J)-$ $\left\{\left(X_{1}, \ldots, X_{t}\right)\right\}$ there is associated a minimal generator $x=\sum_{i=1}^{t} a_{i} e_{i}$ of $M$ with the height of $O_{M}(x)$ at most $r$.

Proof. We note that $r$ is strictly less than $t$ since $M$ is not free. Let $S=$ $R\left[X_{1}, \ldots, X_{t}\right]$ be the full ring of polynomials in $t$ variables over $R$. Let $N$ be the $S$-module $S \otimes_{R} M$ and let $v=\sum_{i=1}^{t} X_{i} e_{i}$. Since the rank of $N$ over $S$ is the same as the rank of $M$ over $R$ and since $v$ is in $\left(X_{1}, \ldots, X_{t}\right) N$, we have from [2,4] that the height of $O_{N}(v)$ is less than or equal to $r$. Let $P$ be a prime ideal of height $r$ containing $O_{N}(v)$. Now the $S$-homomorphisms from $N$ to $S$ are generated over $S$ by the $R$-homomorphisms of $M$ to $R$, and $v$ is a homogeneous element of $N$ of degree one (in the usual grading on $S$ and $N$ ). Consequently $O_{N}(v)$ is a homogeneous ideal of $S$. If $b_{1}, \ldots, b_{d}$ represents a system of parameters of $R$, then the $S$-ideal $P+\mathrm{m} S$ is nilpotent modulo $P+\left(b_{1}, \ldots, b_{d}\right) S$. Thus the height of the ideal $P+\mathfrak{m} S$ is at most $r+\operatorname{dim} R$. But $r+\operatorname{dim} R$ is strictly less than the dimension of $S$ since $r<t$. Hence there are (infinitely many) maximal ideals of the form $\left(\mathfrak{m}, X_{1}-a_{1}, \ldots, X_{t}-a_{t}\right)$ which contain $O_{N}(v)+\mathrm{m} S$. If we let $J$ be the ideal $\left(O_{P}(v)+\mathrm{m} S\right) / \mathrm{m} S$ in $\mathfrak{f}\left[X_{1}, \ldots, X_{t}\right]$, then the maximal ideals in question are simply the preimages of the maximal ideals in $\mathfrak{f}\left[X_{1}, \ldots, X_{t}\right]$ which contain $J$.

Let ( $\left.\mathrm{m}, X_{1}-a_{1}, \ldots, X_{t}-a_{t}\right)$ be such an ideal as above and such that not all the $a_{i}$ are in $\mathrm{m}$. We have that $S /\left(X_{1}-a_{1}, \ldots, X_{t}-a_{t}\right)$ is isomorphic to $R$. Since the $S$-homomorphisms of $N$ to $S$ are generated by the $R$-homomorphisms of $M$ to $R$, the order ideal of the minimal generator $\sum_{i=1}^{t} a_{i} e_{i}$ is

$$
\left(O_{N}(v)+\left(X_{1}-a_{1}, \ldots, X_{t}-a_{t}\right)\right) /\left(X_{1}-a_{1}, \ldots, X_{t}-a_{t}\right)
$$

which has height in $R$ at most $r$ since $S$ is catenary.

We remark here that one should expect that the minimal generators whose order ideals have the correct height be associated to a closed set rather than an open one. Roughly speaking this is because the condition that $O_{N}(v)$ has height too large is a result of the vanishing of certain $\operatorname{Ext}_{S}^{i}\left(O_{N}(v), S\right)$ which is an open condition. Since, as we show later, minimal generators can have order ideals with too large a height, this open set is nonempty and we are left with looking in its complement.

An amusing consequence of the above construction occurs in case the module $M$ represents a vector bundle on the punctured spectrum (i.e., free at all nonmaximal 
primes) of a regular local power series ring $R=\mathfrak{f}\left[\left[T_{1}, \ldots, T_{n}\right]\right]$ where $\mathfrak{f}$ is an algebraically closed field. Assuming that $M$ has rank less than the dimension of $R$, the order ideal $O_{N}(v)$ (notation as in the preceding lemma) has a unique assoicated prime ideal $P$ of height equal to the rank of $M$. To see this we note that $m$ cannot be contained in any associated prime of $O_{N}(v)$ having height less than the dimension of $R$. So there must be $x \in \mathfrak{m}-\mathfrak{m}^{2}$ outside of such a prime ideal. By [3, Theorem 4.1] $M_{z}$ is a free $R_{z}$-module and therefore $N_{z}$ is necessarily a free $S_{z}$-module. Since $e_{1}, \ldots, e_{t}$ generate $N_{z}$, it follows that the order ideal of $v=\sum_{i=1}^{t} X_{i} e_{i}$ is generated by rank $M$ independent polynomial indeterminates in $S_{z}=R_{z}\left[X_{1}, \ldots, X_{t}\right]$. Thus, it follows that $O_{N}(v)$ localized at $z$ is a prime ideal of height equal to $\operatorname{rank}(M)$. Moreover, this is true for any $x \in \mathrm{m}-\mathrm{m}^{2}$. Thus it follows that there must be a unique prime $P$ of height equal to rank $M$ which is associated to $O_{N}(v)$ in $S$. We do not know if this uniqueness can be maintained after specializing the variables $X_{1}, \ldots, X_{t}$.

2. Special cases. There are at least two distinct ways of producing minimal generators whose order ideals have height exceeding the rank of the module. Let $(R, \mathfrak{m})$ be a regular local ring of dimension at least four. Let $E$ be a reflexive nonfree $R$-module of rank two which is free on the punctured spectrum of $R$. Then it follows from [5, Theorem 2.4] that $\operatorname{Ext}^{1}(E, R)$ is nonzero. For a definite example one may take the Horrocks and Mumford bundle of rank two on $\mathbf{P}^{4}$ whose cohomology is explicitly computed [7]. Let $\xi$ in $\operatorname{Ext}^{1}(E, R)$ be a nonzero element and let $0 \rightarrow R \rightarrow M \rightarrow E \rightarrow 0$ be an extension corresponding to $\xi$. Let $x$ be the image of 1 in $M$. Then the annihilator of $\xi$ is $O_{M}(x)$. But $E$ is free on the punctured spectrum of $R$ and so the annihilator of $\xi$ is m-primary. Thus $M$ has rank three while the height of $O_{M}(x)$ is $\operatorname{dim} R$ which is at least four. It follows from [4] that $x$ is necessarily a minimal generator.

The second method of producing minimal generators with large order ideals uses Gulliksen's construction [6] (see also [1]) of three generated ideals $(a, b, c)$ in a regular local ring $R$ such that the depth of $R /(a, b, c)$ is zero while the dimension of $R$ can be made arbitrary. Since $R /(a, b, c)$ has depth zero, there exists a $d$ not in $(a, b, c)$ such that $m d$ is contained in $(a, b, c)$. Let $N=R^{4} /\langle a, b, c, d\rangle R$. Let $x$ be the image of $\langle 0,0,0,1\rangle$ in $N$. Then the relation $m d \subseteq(a, b, c)$ translates to the existence of homomorphisms of $N$ to $R$ which carry $x$ to any prescribed element of m. Thus $N$ has rank three while $O_{N}(x)=\mathfrak{m}$ has height equal to the dimension of $R$.

The fact that our examples are modules of rank at least three is not a coincidence. The following elementary result explains why.

Proposition. Let $R$ be a regular local ring and let $M$ be an $R$-module of rank one or two. If $x \in M$ has order ideal whose height exceeds the rank of $M$, then $x$ generates a free summand of $M$.

Proof. We may assume that $M$ is torsion free. If $M$ has rank one, then $M$ is isomorphic to an ideal of $R$. If $M$ is isomorphic to $R$ and if $x$ goes to $a \in R$ under this isomorphism, then $O_{M}(x)$ is the ideal generated by $a$. If that ideal is proper, it is of height one. Otherwise $M$ is isomorphic to a proper ideal $I$ in $R$ of height at least 
two. But then $\operatorname{Hom}(I, R)$ is induced by multiplication. Thus $O_{M}(x)$ is inside a proper principal ideal.

Now suppose that $M$ has rank two and that $O_{M}(x)$ has height at least three. Since the homomorphisms of $M^{* *}$ to $R$ are the same as those of $M$ to $R$ we may replace $M$ with $M^{* *}$, that is, we may assume that $M$ is reflexive and hence a second syzygy. Moreover $x$ is a minimal generator of $M$ by [2, 4]. However, from Bruns [1] the module $M / R x$ is again a second syzygy. But that makes $M / R x$ a reflexive ideal and therefore isomorphic to $R$. Thus $M$ is isomorphic to $R \oplus R$, where $x$ is one of the minimal generators. It follows that $O_{M}(x)$ is $R$ itself. Thus $x$ generates a summand as desired.

\section{REFERENCES}

1. W. Bruns, "Jede" endliche freie Auflösung ist freie Auflösung eines von drei Elementen erzeugten Ideals, J. Algebra 39 (1976), 429-439.

2. The Eisenbud-Evans generalized principal ideal Theorem and determinantal ideals, Proc. Amer. Math. Soc. 83 (1981), 19-24.

3. W. Bruns, E. G. Evans and P. Griffith, Syzygies, ideals of height two, and vector bundles, J. Algebra 67 (1980), 143-162.

4. D. Eisenbud and E. G. Evans, A generalized principal ideal theorem, Nagoya Math. J. 62 (1976), $41-53$.

5. E. G. Evans and P. Griffith, The syzygy problem, Ann. of Math. 114 (1981), 323-333.

6. T. Gulliksen, Tout ideal premier d'un anneau noethérien est associé à une ideal engendré par trois éléments, C. R. Acad. Sci. Paris Sér. A 271 (1970), 1207-1208.

7. G. Horrocks and D. Mumford, A rank 2 bundle on $\mathbf{P}^{4}$ with 15,000 symmetries, Topology 12 (1973), $63-81$.

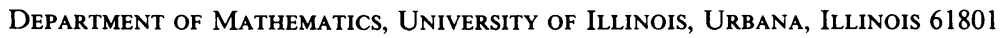

